\title{
Efficacité et innocuité des écrans solaires pour la prévention du cancer de la peau
}

\author{
Megan Sander MD, Michael Sander DMD, Toni Burbidge MD, Jennifer Beecker MD
}

Citation : CMAJ 2020 December 14;192:E1802-8. doi : 10.1503/cmaj.201085-f

Voir la version anglaise de l'article ici : www.cmaj.ca/lookup/doi/10.1503/cmaj.201085

$\mathbf{P}$ lus de 80000 cas de cancer de la peau sont diagnostiqués au Canada chaque année ${ }^{1}$. Étant donné qu'on estime à 80\%-90\% la proportion de ces cancers qui sont associés à une exposition aux rayons ultraviolets, on préconise l'utilisation d'écrans solaires qui bloquent les rayons ultraviolets comme importante mesure de prévention des cancers de la peau ${ }^{2,3}$, des coups de soleil et du photovieillissement cutané (voir définitions à l'annexe 1 , accessible en anglais au www.cmaj.ca/lookup/doi/10.1503/ cmaj.201085/tab-related-content). Il a été démontré que les écrans solaires réduisent l'incidence du mélanome et des cancers de la peau non mélaniques ${ }^{4,5}$. Tant l'Association canadienne de dermatologie que l'American Academy of Dermatology recommandent l'utilisation d'écrans solaires pour la prévention du cancer de la $p^{p e a u^{6,7}}$. Or, depuis la mise au point du premier des écrans solaires commerciaux en 1928, on s'interroge sur l'innocuité et l'efficacité de ces derniers, et plus récemment, on s'est inquiété de leurs répercussions environnementales. Nous résumons ici les données probantes relatives à l'efficacité et aux préjudices associés aux écrans solaires pour aider les médecins à bien conseiller leurs patients (encadré 1 ).

\section{Comment les écrans solaires agissent-ils?}

Les écrans solaires contiennent des substances chimiques (organiques) ou minérales (physiques/inorganiques) qui agissent en bloquant les rayons ultraviolets dont la longueur d'onde est plus courte que celle de la lumière visible (subdivisés en rayons ultraviolets $A$ [UVA]1, UVA2, B [UVB] et C [UVC]), comme l'illustre la figure 1. En

\footnotetext{
Encadré 1 : Données utilisées pour la présente revue

Nous avons procédé à une interrogation ciblée du réseau MEDLINE à partir d'une combinaison des mots clés en langue anglaise " sunscreen », «skin cancer », « melanoma », « squamous cell carcinoma », « basal cell carcinoma », « photoaging », « safety » et «environment » afin de dégager les études publiées entre 1984 et 2020. Nous avons plus spécifiquement cherché des essais randomisés et contrôlés, des revues systématiques et des méta-analyses portant sur les questions cliniques de cet article. Nous avons aussi identifié les articles de synthèse, les publications en sciences fondamentales et les lignes directrices institutionnelles. Nous avons complété notre recherche en consultant les articles de nos propres collections.
}

\section{POINTS CLÉS}

- Plusieurs essais randomisés et contrôlés rigoureux comportant des suivis à long terme ont montré que les écrans solaires réduisent le risque de carcinome épidermoïde et de mélanome.

- Les écrans solaires commerciaux protègent la peau contre les effets nocifs des rayons ultraviolets grâce à leurs ingrédients chimiques ou minéraux.

- L'Association canadienne de dermatologie recommande l'utilisation d'une quantité adéquate d'écran solaire à large spectre doté d'un facteur de protection solaire d'au moins 30 pour la plupart des enfants et des adultes dans le cadre d'une stratégie de photoprotection complète.

- Selon des données émergentes, certains ingrédients des écrans solaires chimiques sont absorbés par voie systémique, mais la portée clinique de ce phénomène reste à définir; il faudra approfondir la recherche pour vérifier si cela est préjudiciable.

- Les filtres ultraviolets des écrans solaires chimiques pourraient être nocifs pour l'environnement.

général, la longueur d'onde des rayons est inversement proportionnelle à leur nocivité pour la peau. Les filtres des écrans solaires sont efficaces contre les UVA1, les UVA2 et les UVB. Les filtres chimiques, comme l'oxybenzone, l'avobenzone, l'octocrylène et l'écamsule, sont des composés aromatiques qui absorbent les rayons ultraviolets de haute intensité et les convertissent en chaleur (état excité suivi d'une production d'énergie). Quand ces molécules reviennent à leurs niveaux de base, il en résulte une conversion de l'énergie absorbée en des longueurs d'onde de plus faible énergie, comme le rayonnement infrarouge (c.-à-d., chaleur) ${ }^{8}$.

Les filtres des écrans minéraux, comme le dioxyde de titane et l'oxyde de zinc, protègent la peau en réfléchissant ou en réfractant les rayons ultraviolets; par contre, des études expérimentales ont montré que si les particules sont de très petite taille, comme dans les écrans solaires micronisés, le mode d'action est comparable à celui des filtres chimiques. Plus spécifiquement, l'oxyde de zinc et le dioxyde de titane micronisés se comportent comme des métaux semi-conducteurs, qui absorbent la lumière ultraviolette dans la majeure partie du spectre électromagnétique ${ }^{9}$. Une liste des ingrédients des écrans solaires actuellement approuvés par Santé Canada se trouve au tableau $1^{10}$. 

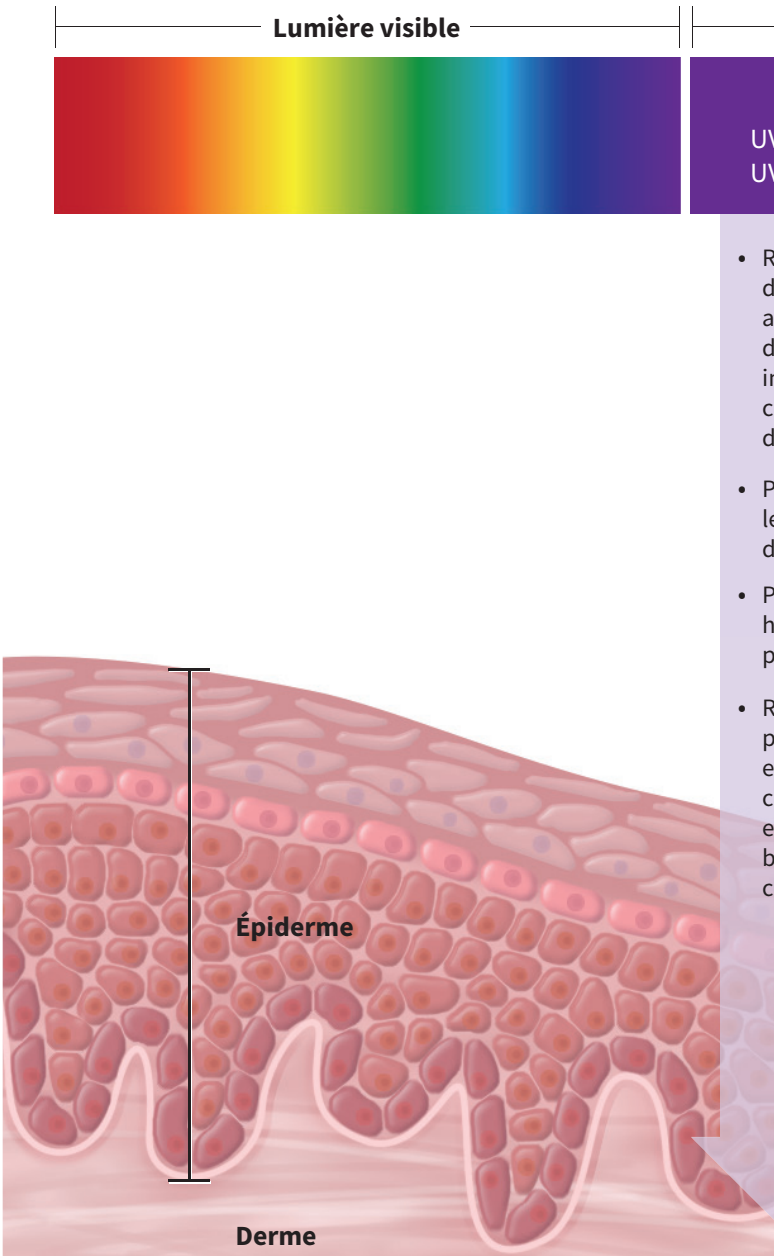

Lumière ultraviolette

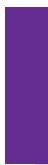

UVA

UVA1 : 300-400 nm

UVA2 : $315-340 \mathrm{~nm}$

- Représentent $95 \%$ des RUV qui atteignent la surface de la terre avec une intensité assez constante au cours de la journée

- Pénètrent l'ozone, les nuages et la vitre des fenêtres

- Pénètrent la peau humaine plus en profondeur

- Responsables du photovieillissement et de la carcinogenèse, et moins du bronzage et des coups de soleil

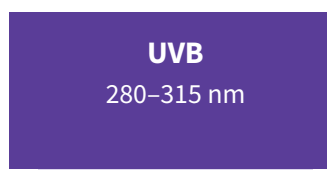

- Représentent $5 \%$ des RUV qui de la terre avec une intensité variable qui culmine au milieu de la journée

- Partiellement absorbés par l'ozone, les nuages et ne pénètrent pas la vitre des fenêtres

- Non absorbés aussi profondément dans la peau que les UVA

- Principalement responsables du bronzage et des coups de soleil et moins du photovieillissement et de la carcinogenèse atteignent la surface

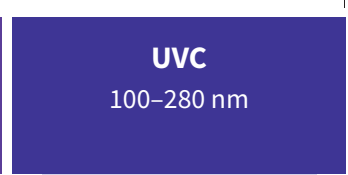

- Représentent moins de $1 \%$ des RUV qui atteignent la surface de la terre

- Non absorbés profondément dans la peau

- Absorbés par l'atmosphère et la couche d'ozone

- Germicide

Figure 1 : Représentation schématique du spectre électromagnétique de la lumière, avec rappel des fréquences des rayons ultraviolets (RUV) et leur effet sur la peau humaine. En général, la longueur d'onde des rayons est inversement proportionnelle à leur nocivité au plan biologique. Remarque : UVA = ultraviolet $\mathrm{A}, \mathrm{UVB}=$ ultraviolet $\mathrm{B}, \mathrm{UVC}=$ ultraviolet $\mathrm{C}$. Les filtres des écrans solaires bloquent les rayons UVA1, UVA2 et UVB.

\section{Quelle est l'efficacité des écrans solaires pour la prévention du photovieillissement et du cancer de la peau?}

Selon des données probantes tirées d'études d'observation ${ }^{11}$, d'un volumineux essai randomisé et contrôlé (ERC) ${ }^{12}$ et de petites études expérimentales non randomisées ${ }^{13-15}$, les écrans solaires préviendraient efficacement les signes de photovieillissement, y compris les rides, la télangiectasie et les anomalies pigmentaires induites par les rayons ultraviolets ${ }^{11-15}$. Malgré les difficultés auxquelles se bute la recherche sur le cancer de la peau, en raison de sa pathogenèse multifactorielle et de son évolution parfois lente, les données suivantes appuient le recours aux écrans solaires pour la prévention du cancer de la peau.

Des études expérimentales menées dans les années 1980 et 1990 ont montré que les écrans solaires protègent contre les lésions cellulaires associées à la carcinogenèse dans des modèles animaux ${ }^{16,17}$. Dans un ERC communautaire rigoureux de 4,5 ans regroupant 1621 adultes australiens, avec un suivi de plus d'une dizaine d'années, l'incidence des carcinomes épidermoïdes était inférieure de $40 \%$ chez les participants assignés aléatoirement au groupe soumis à une utilisation quotidienne d'écrans solaires, comparativement aux participants qui les utilisaient à leur discrétion (rapport des taux 0,61, intervalle de confiance [IC] à 95\% 0,46$0,81)^{4,18}$. Toutefois, l'incidence du carcinome basocellulaire n'a pas significativement diminué, peut-être en raison de sa pathogenèse lente ${ }^{18}$. Près de 15 ans après la fin de l'étude, les participants qui ont utilisé des écrans solaires quotidiennement pendant la période de 4,5 ans de l'étude ont présenté un risque notablement moindre de mélanome invasif (rapport de risque [HR] 0,27, IC à $95 \% 0,08$ à -0,97), même si très peu de mélanomes invasifs ont été enregistrés, compte tenu du temps que prend cette tumeur à se développer ${ }^{5}$. Une analyse de sous-groupes prédéfinis dans le cadre de cet essai a confirmé que l'utilisation régulière des écrans solaires sur une période de 4,5 ans peut stopper les signes de vieillissement cutané causés par l'exposition au solei ${ }^{12}$. Un autre volumineux ERC australien a fait état d'un taux significativement moindre de cas de kératose actinique (précurseur du carcinome 
Tableau 1 : Ingrédients des écrans solaires approuvés par Santé Canada ${ }^{10}$

\begin{tabular}{|c|c|c|c|}
\hline Ingrédient médicinal & Autres noms & Protection UV & $\begin{array}{c}\text { Ingrédient } \\
\text { médicinal, \% }\end{array}$ \\
\hline Dioxyde de titane & Aucun & UVA 2 & $\leq 25$ \\
\hline Oxyde de zinc & Aucun & UVA 1 & $\leq 25$ \\
\hline Acide para-aminobenzoïque & Aucun & UVB & $\leq 15$ \\
\hline \multirow[t]{2}{*}{ Avobenzone } & Butyl méthoxydibenzoylméthane & UVA 1 & $\leq 3$ \\
\hline & Parsol 1789 & UVB & \\
\hline Ensulizole & Acide 2-phénylbenzimidazole-5-sulfonique & UVB & $\leq 4$ \\
\hline Homosalate & Homomenthylsalicylate & UVB & $\leq 15$ \\
\hline \multirow[t]{2}{*}{ Méradimate } & 2-aminobenzoate de méthyle & UVA 2 & $\leq 5$ \\
\hline & Anthranilate de méthyle & & \\
\hline \multirow[t]{2}{*}{ Octinoxate } & Méthoxycinnamate de 2-éthylhexyle & UVB & $\leq 7,5$ \\
\hline & Méthoxycinnamate d'octyle & & \\
\hline \multirow[t]{2}{*}{ Octisalate } & Salicylate de 2-éthylhexyle & UVB & $\leq 5$ \\
\hline & Salicylate d'octyle & & \\
\hline Octocrylène & 2-Éthylhexyl 2-cyano-3,3-diphénylacrylate & UVA 2 & $\leq 10$ \\
\hline \multirow[t]{2}{*}{ Oxybenzone } & Benzophénone-3 & UVA 2 & $\leq 6$ \\
\hline & 2-Hydroxy-4-méthoxybenzophénone & UVB & \\
\hline Sulisobenzone & Benzophénone-4 & UVA 2 & $\leq 10$ \\
\hline Drométrizole trisiloxane & Méxoryl XL & UVA & $\leq 15$ \\
\hline Enzacamène & 4-Méthylbenzylidène camphre & UVB & $\leq 6$ \\
\hline \multirow[t]{2}{*}{ Padimate-O } & Octyl diméthyl PABA & UVB & $\leq 8$ \\
\hline & $\sigma-\mathrm{PABA}$ & & \\
\hline \multirow{4}{*}{$\begin{array}{l}\text { Acide téréphthalylidène dicamphre } \\
\text { sulfonique }\end{array}$} & Méxoryl SX & UVA & $\leq 10$ \\
\hline & 3,3'-(1,4-phénylènediméthylidène) & UVB & \\
\hline & $\begin{array}{l}\text { Acide bis(7,7-diméthyl-2-oxobicyclo[2.2.1] hept-1-yl } \\
\text { méthanesulfonique) }\end{array}$ & & \\
\hline & Écamsule & & \\
\hline Cinoxate & 2-Éthoxyéthyl 3-(4-méthoxyphényl) propanoate & UVA & $\leq 3$ \\
\hline Diéthanolamine-méthoxycinnamate & Aucun & UVB & $\leq 10$ \\
\hline \multirow[t]{2}{*}{ Dioxybenzone } & Benzophénone-8 & UVA & $\leq 3$ \\
\hline & $\begin{array}{l}\text { (2-Hydroxy-4-méthoxyphényl)(2-hydroxyphényl) } \\
\text { méthanone }\end{array}$ & UVB & \\
\hline Salicylate de triéthanolamine & Salicylate de trolamine & UVB & $\leq 12$ \\
\hline
\end{tabular}

épidermoïde) chez les participants assignés à l'utilisation régulière des écrans solaires, comparativement aux témoins qui ont utilisé une crème à ingrédients inertes pendant un été (rapport des taux $0,62$, IC à $95 \% 0,54$ à $-0,71)^{19}$.

Chez les bénéficiaires d'une greffe d'organe, une population à haut risque de morbidité et de mortalité par cancer de la peau, une étude prospective menée dans un seul centre a porté sur 120 patients appariés et a montré que l'utilisation d'écrans solaires dotés d'un facteur de protection solaire (FPS) de 50 sur une période de 24 mois réduisait l'apparition de la kératose actinique, du carcinome épidermoïde et dans une moindre mesure, du carcinome basocellullaire ${ }^{20}$. De récentes méta-analyses n'ont pas corroboré les conclusions de ces ERC; elles n'ont pas observé d'efficacité notable des écrans solaires à prévenir le mélanome ou les cancers de la peau non mélaniques ${ }^{21,22}$. Par contre, ces méta-analyses incluaient des études rétrospectives comportant des disparités méthodologiques, et une incluait des études n'ayant porté que sur les filtres antiUVB (plutôt que des écrans solaires à large spectre) ${ }^{21}$. Dans l'ensemble, les données probantes de meilleure qualité disponibles laissent entendre que les écrans solaires préviennent effectivement le cancer de la peau. 


\section{Qui devrait utiliser des écrans solaires?}

L'American Academy of Dermatology recommande l'utilisation régulière d'écrans solaires à FPS 30 ou plus pour tous, peu importe le type de peau ${ }^{23}$, même si le cancer de la peau est beaucoup plus prévalent chez les personnes à la peau blanche que chez les personnes à la peau foncée ${ }^{24}$. Aucune étude n'a mesuré l'efficacité de l'utilisation régulière des écrans solaires à réduire le risque de cancer de la peau chez les personnes non blanches.

Chez les enfants de 6 mois et plus, tout comme chez les adultes, l'Association canadienne de dermatologie recommande l'utilisation d'écrans solaires à large spectre dotés d'un FPS de 30 ou plus ${ }^{7}$. Des études sur l'application d'un écran solaire à FPS 100 sur une moitié du visage et FPS 50 sur l'autre, ont montré que les écrans solaires à FPS 100 sont les plus efficaces à prévenir les coups de soleil dans des conditions normales d'utilisation, sur la plage ${ }^{25}$ ou les pistes de ski en haute altitude ${ }^{26}$.

Santé Canada ne recommande pas les écrans solaires chez les enfants de moins de 6 mois, en raison d'un risque théorique d'absorption accrue de leurs ingrédients résultant

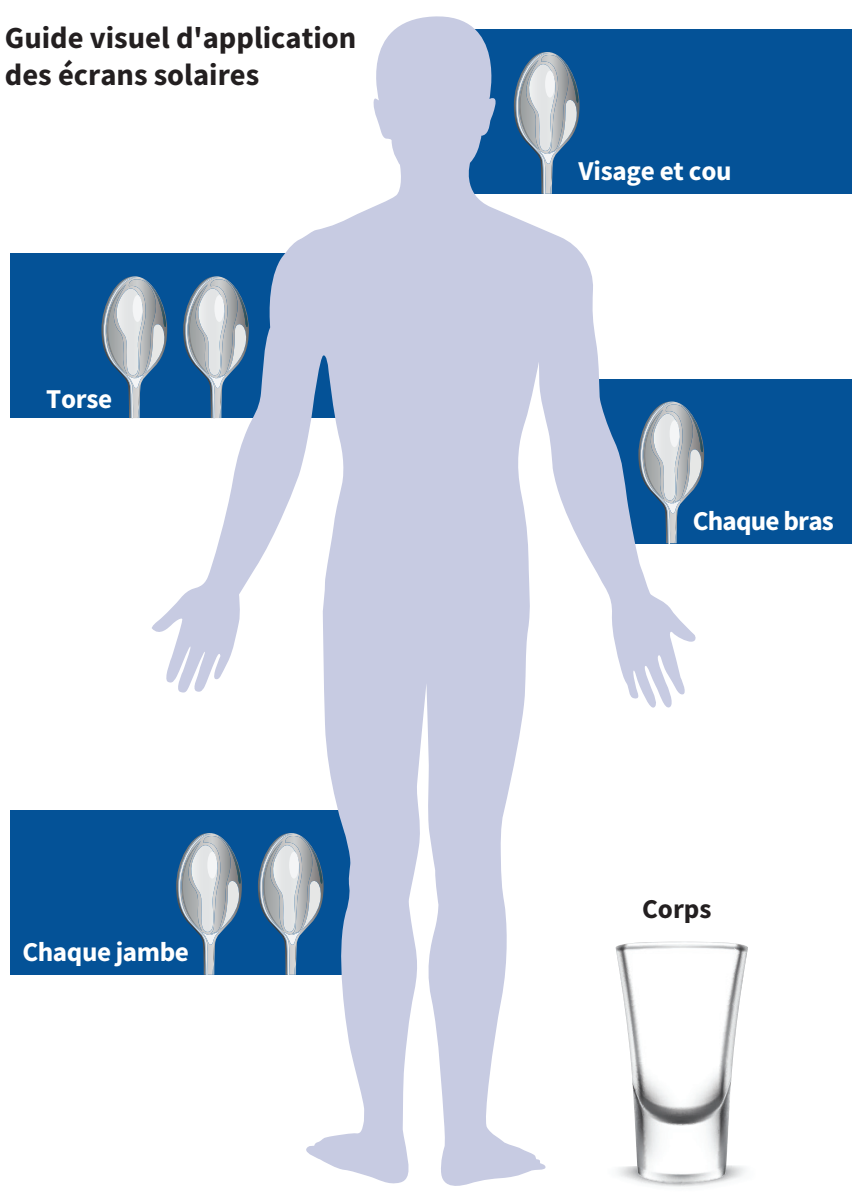

Figure 2 : Guide visuel d'application de l'écran solaire pour une personne de gabarit moyen, selon les recommandations de la Société canadienne du cancer et de l'American Academy of Dermatology. d'un rapport surface corporelle:volume plus élevé et d'un épiderme plus mince ${ }^{27}$. Les règles principales pour protéger les bébés contre le soleil consistent à ne pas les exposer et à utiliser des vêtements protecteurs ${ }^{28}$. Si on applique un écran solaire sur la peau d'un bébé, les experts conseillent de le laver le plus rapidement possible dès qu'il n'est plus requis ${ }^{29}$ et de privilégier les écrans solaires minéraux plutôt que les écrans solaires chimiques.

\section{Comment doit-on appliquer les écrans solaires?}

Selon des études d'observation, les consommateurs appliquent généralement trop peu d'écran solaire, l'utilisation standard se situant à 20\%-50\% de la quantité recommandée ${ }^{30-32}$. Par contre, l'utilisation d'écrans solaires à FPS plus élevé pourrait compenser l'application insuffisante ${ }^{26}$. Par exemple, si un écran solaire FPS 50 est appliqué dans des conditions réelles, il pourrait équivaloir à un FPS 25 .

Lors d'une réunion consensuelle canadienne de 2015, le libellé " appliquer l'écran solaire généreusement » a été jugé le plus approprié en raison des différences de gabarit des utilisateurs ${ }^{33}$. La figure 2 offre une estimation grossière de la quantité d'écran solaire qu'une personne de gabarit moyen devrait appliquer, de l'avis de la Société canadienne du cancer et de l'American Academy of Dermatology.

Même si l'étiquette des produits mentionne souvent que les écrans solaires doivent être appliqués 15-30 minutes avant une sortie à l'extérieur ${ }^{34}$, selon une récente étude, la protection contre les rayons ultraviolets est immédiate dès l'application, mais la protection n'a pas été revérifiée après la baignade ou un épisode de transpiration ${ }^{35}$. Il serait donc prudent d'attendre 15-30 minutes si on a besoin d'une protection hydrofuge.

De récentes études expérimentales ont montré que les écrans solaires persistent sur la peau avec le FPS désiré jusqu'à 8 heures après une seule application ${ }^{35-38}$; on en déduit qu'il n'est plus obligatoire de suivre le conseil de réappliquer l'écran solaire toutes les 2-3 heures si les personnes sont physiquement actives. On suggère toutefois la réapplication si l'écran solaire risque fort d'avoir été éliminé par la transpiration, la baignade, la friction contre les vêtements ou l'exfoliation par le sable ${ }^{39-41}$. Pour la baignade ou en cas de transpiration, il faut utiliser des écrans solaires hydrofuges ${ }^{40}$.

Les écrans solaires en vaporisateurs sont moins intéressants que les écrans solaires en crème, et ce, pour diverses raisons. Le vent peut disperser l'écran solaire en vaporisateur, l'application sera alors insuffisante. En outre, souvent, comme les écrans solaires en vaporisateur sèchent rapidement et sont pratiquement invisibles sur la peau, il est difficile de vérifier si l'application est uniforme ${ }^{42}$. Les écrans solaires en aérosol sont aussi inflammables et plusieurs cas de brûlures cutanées ont été signalés après une exposition à des flammes nues, même après qu'on ait laissé sécher les écrans solaires. Somme toute, les risques d'inhalation des écrans solaires en aérosols n'ont pas fait l'objet d'études adéquates ${ }^{43}$. 


\section{Quels sont les principaux problèmes d'innocuité?}

\section{Réactions cutanées}

Les réactions indésirables les plus souvent associées aux écrans solaires sont une irritation subjective (p. ex., sensation de picotements et de brûlure) sans érythème, la dermatite de contact irritative et la comédogénicité. Plus rarement, les ingrédients chimiques des écrans solaires peuvent aussi déclencher une dermatite de contact allergique et une dermatite de contact photoallergique; les ingrédients allergéniques le plus souvent incriminés sont l'octocrylène, l'oxybenzone et l'octyl-méthoxycinnamate ${ }^{44}$.

\section{Absorption des écrans solaires}

En 2019, un petit ERC regroupant 24 participants commandité par la Food and Drug Administration des États-Unis (FDA) a noté une absorption systémique de 4 ingrédients des écrans solaires : l'oxybenzone, l'avobenzone, l'octocrylène et l'écamsule ${ }^{45}$. Appliqués dans des conditions d'utilisation maximale pendant 4 jours consécutifs, ces composés ont atteint des taux sanguins qui excédaient les limites recommandées dans les lignes directrices de la FDA ${ }^{45}$. Les investigateurs ont de plus noté de longues demivies pour chacun de ces ingrédients, donnant à penser que l'utilisation régulière des écrans solaires pourrait entraîner une accumulation dans l'organisme ${ }^{46}$. Une étude de suivi a confirmé ces observations ${ }^{47}$. La plupart des gens utilisent cependant une quantité bien moindre d'écran solaire et malgré leurs conclusions, les investigateurs de l'étude ont préconisé l'utilisation des écrans solaires en raison de leurs effets protecteurs éprouvés, étant donné qu'on ignore encore la portée clinique de l'absorption de ces ingrédients. Il faudra approfondir la recherche pour déterminer si l'absorption des ingrédients des écrans solaires peut avoir des conséquences négatives sur la santé.

Contrairement aux ingrédients des écrans solaires chimiques, ceux des écrans solaires minéraux ne sont pas absorbés par voie systémique. Une étude in vitro a révélé que moins de 0,03\% des nanoparticules de zinc ont pénétré la couche cornée supérieure et aucune particule n'a été détectée dans la couche cornée inférieure ${ }^{48}$. Les écrans solaires minéraux ont de tout temps semblé moins attrayants que les écrans solaires chimiques, car ils laissent un résidu blanc sur la peau, et pourraient de ce fait être appliqués en quantité insuffisante. Les améliorations apportées aux préparations et la micronisation des filtres minéraux anti-UV ont permis de mettre au point des écrans solaires minéraux plus acceptables visuellement ${ }^{49}$.

\section{Effets endocriniens}

Des données de faible qualité ont soulevé la question de possibles effets œstrogéniques et antiandrogéniques des écrans solaires chimiques. Même si une récente méta-analyse a fait état d'un lien entre l'oxybenzone et des effets négatifs sur l'appareil reproducteur des poissons, la littérature résumée était hétérogène et par conséquent, les résultats étaient peu concluants ${ }^{50}$. Chez des sujets humains, une étude prospective a mentionné une baisse de la fertilité chez des hommes ayant été exposés à la benzophénone-2 et à la 4-hydroxybenzophénone, mais ces observations pourraient être dues à des variables de confusion propres à l'étude ${ }^{51}$. Une revue systématique qui évaluait des études chez l'animal et chez l'humain, a mentionné que des taux élevés d'exposition à l'oxybenzone durant la grossesse étaient associés à une diminution de l'âge gestationnel chez les nouveau-nés de sexe masculin et à des poids à la naissance plus bas chez les nouveau-nés de sexe féminin ${ }^{50}$. Une forte hétérogénéité a toutefois limité l'utilité de cette étude ${ }^{50}$.

\section{Comment les écrans solaires affectent-ils l'environnement?}

De récentes études ont signalé que des ingrédients des écrans solaires chimiques sont détectables dans diverses sources hydriques ${ }^{52,53}$ et pourraient persister en dépit du traitement de l'eau ${ }^{54}$. Se rajoute depuis peu, le problème de la détection des filtres des écrans solaires dans les tissus de différentes espèces de poisson, ce qui soulève le risque de bioaccumulation et de bioamplification ${ }^{55}$.

Les effets des ingrédients des écrans solaires sur les récifs de corail suscitent présentement l'intérêt de la recherche scientifique. Des études in vitro ont montré que l'oxybenzone affecte les larves présentes dans les récifs corallien ${ }^{56}$ et contribueraient au phénomène de blanchiment de ces derniers. Il faut néanmoins tenir compte d'autres variables de confusion, notamment la hausse de la salinité et de la température des océans associée au réchauffement climatique ${ }^{55}$. Ces études préliminaires ont conduit au bannissement de l'oxybenzone et de l'octinoxate dans certaines régions ${ }^{57}$.

\section{Quelles autres mesures photoprotectrices peut-on recommander?}

Les écrans solaires ne sont qu'un élément dans une stratégie de photoprotection complète. Il est important de conseiller les patients au sujet des mesures de protection contre les rayons ultraviolets, soit porter des chapeaux à large bord, protéger ses yeux (p. ex., verres fumés enveloppants munis de protection antiUV) et chercher l'ombre lorsque l'indice UV est supérieur à 3 (habituellement de $11 \mathrm{~h}$ à $15 \mathrm{~h}$, d'avril à septembre au Canada) $)^{33}$. En général, les vêtements faits d'étoffes épaisses, tissées serrées, comme le polyester et le coton ou le nylon et l'élastane (p. ex., Spandex, Lycra) et les couleurs sombres offrent une meilleure protection ${ }^{58,59}$. Des vêtements ont été conçus pour protéger contre le soleil, avec un facteur de protection anti-UV allant jusqu'à $50^{28}$. Tous les vêtements protègent moins s'ils sont mouillés ou étirés ${ }^{59}$.

\section{Nouvelles technologies potentiellement photoprotectrices}

Les photolyases et les antioxydants topiques (vitamine C, vitamine $E$, sélénium et polyphénols présents dans les extraits de thé vert) sont perçus comme de potentiels agents photoprotecteurs émergents, par voie topique ou autre. Il n'est pas encore possible de stabiliser les antioxydants dans des préparations d'écrans solaires de manière à ce qu'ils restent biologiquement actifs. Des études ont conclu que les écrans solaires prétendument dotés d'une activité antioxydante en sont pour ainsi dire dépourvus ${ }^{60-62}$. 
Des agents photoprotecteurs administrés par voie orale, comme la niacinamide et l'extrait de Polypodium leucotomos, dérivé d'une fougère des Amérique centrale et du Sud, sont utilisés pour prévenir les signes de vieillissement cutané causés par l'exposition au soleil. De petits ERC ont révélé que l'extrait de $P$. leucotomos permet d'augmenter la dose d'érythème minimal lors de tests d'exposition au soleil, sans effets indésirables notables, en plus d'être utiles pour les maladies dermatologiques induites par les rayons ultraviolets, comme la lucite polymorphe et l'urticaire solaire ${ }^{63-65}$.

Le nicotinamide, aussi appelé niacinamide, est la forme amide active de la niacine (vitamine B3), mais contrairement à la niacine, il ne cause pas de rougeurs cutanées. Dans des études antérieures, on a démontré que le nicotinamide favorise la réparation de l'ADN et diminue la formation de dimères de cyclobutanepyrimidine dans les kératinocytes humains ${ }^{62}$. Dans un ERC de phase III, qui n'a pas été répliqué, à raison de $500 \mathrm{mg} 2$ fois par jour, le nicotinamide a été associé à un taux moindre de cas de kératose actinique et de cancer de la peau non mélanique sur une période de 12 mois $^{66}$. Par contre, les cancers de la peau qui sont survenus ont eu tendance à être de haut grade.

\section{Conclusion}

L'exposition aux rayons ultraviolets a des effets nocifs directs et a été associée au cancer de la peau, fréquent au Canada. Des données probantes de grande qualité ont montré que les écrans solaires réduisent le risque de mélanome et de cancer de la peau non mélanique. Les médecins devraient par conséquent, conseiller leurs patients au sujet des stratégies de photoprotection, y compris, éviter le soleil du midi, chercher l'ombre et porter des vêtements protecteurs, en plus d'appliquer un écran solaire s'il est impossible d'éviter l'exposition au soleil. À l'heure actuelle, pour la photoprotection, l'Association canadienne de dermatologie recommande l'utilisation d'écrans solaires à large spectre dotés d'un FPS d'au moins 30 à partir de l'âge de 6 mois. Des données de moins bonne qualité ont montré que certains ingrédients des écrans solaires chimiques sont absorbés par l'organisme et pourraient polluer l'environnement; les personnes qui se soucient de l'environnement pourraient opter pour des écrans solaires minéraux comme solution de rechange. La recherche sur l'innocuité et l'efficacité des écrans solaires sur des agents novateurs se poursuit.

\section{Références}

1. Canadian Cancer Society's Advisory Committee on Cancer Statistics. Canadian cancer statistics 2014: special topic: skin cancers. Canadian Cancer Statistics. Toronto: Canadian Cancer Society; 2014;1-132. Accessible ici : www.cancer. $\mathrm{ca} /$ /media/cancer.ca/CW/cancer information/cancer 101/Canadian cancer statistics/Canadian-Cancer-Statistics-2014-EN.pdf (consulté le 15 mars 2020).

2. Koh HK, Geller AC, Miller DR, et al. Prevention and early detection strategies for melanoma and skin cancer. Current status. Arch Dermatol 1996;132:436-43.

3. Parkin DM, Mesher D, Sasieni P. 13. Cancers attributable to solar (ultraviolet) radiation exposure in the UK in 2010. Br J Cancer 2011;105(Suppl 2):S66-9.

4. Green A, Williams G, Neale R, et al. Daily sunscreen application and betacarotene supplementation in prevention of basal-cell and squamous-cell carcinomas of the skin: a randomised controlled trial. Lancet 1999;354:723-9.

5. Green AC, Williams GM, Logan V, et al. Reduced melanoma after regular sunscreen use: randomized trial follow-up. J Clin Oncol 2011;29:257-63.
6. Prevent skin cancer. Schaumburg (IL): American Academy of Dermatology; 2016:1. Accessible ici : www.aad.org/public/diseases/skin-cancer/prevent/how (consulté le 15 mars 2020).

7. Canadian Dermatology Association position statement sun protection and sunscreen use. Ottawa: Canadian Dermatology Association; 2020. Accessible ici : dermatology.ca/wp-content/uploads/2020/02/Sun-Protection-and-Sunscreen -Use-Position-Statement-EN.pdf (consulté le 15 mars 2020).

8. Gasparro FP, Mitchnick M, Nash JF. A review of sunscreen safety and efficacy. Photochem Photobiol 1998;68:243-56.

9. Geoffrey K, Mwangi AN, Maru SM. Sunscreen products: rationale for use, formulation development and regulatory considerations. Saudi Pharm J 2019; 27:1009-18.

10. Draft: guidance document - sunscreen monograph. Ottawa: Health Canada; 2012. Accessible ici : www.canada.ca/en/health-canada/services/drugs -health-products/public-involvement-consultations/natural-health-products/ draft-guidance-document-sunscreen-monograph-consultation-document.html (consulté le 10 mars 2020).

11. Boyd AS, Naylor M, Cameron GS, et al. The effects of chronic sunscreen use on the histologic changes of dermatoheliosis. J Am Acad Dermatol 1995;33:941-6.

12. Hughes MCB, Williams GM, Baker $P$, et al. Sunscreen and prevention of skin aging: a randomized trial. Ann Intern Med 2013;158:781-90.

13. Phillips TJ, Bhawan J, Yaar M, et al. Effect of daily versus intermittent sunscreen application on solar simulated UV radiation-induced skin response in humans. J Am Acad Dermatol 2000;43:610-8.

14. Seité S, Reinhold K, Jaenicke T, et al. Broad-spectrum moisturizer effectively prevents molecular reactions to UVA radiation. Cutis 2012;90:321-6.

15. Iannacone MR, Hughes MCB, Green AC. Effects of sunscreen on skin cancer and photoaging. Photodermatol Photoimmunol Photomed 2014;30:55-61.

16. Sambuco CP, Forbes PD, Davies RE, et al. An animal model to determine sunscreen protectiveness against both vascular injury and epidermal cell damage. J Am Acad Dermatol 1984;10:737-43.

17. Ananthaswamy HN, Loughlin SM, Cox P, et al. Sunlight and skin cancer: inhibition of p53 mutations in UV-irradiated mouse skin by sunscreens. Nat Med 1997;3:510-4.

18. van der Pols JC, Williams GM, Pandeya N, et al. Prolonged prevention of squamous cell carcinoma of the skin by regular sunscreen use. Cancer Epidemiol Biomarkers Prev 2006;15:2546-8.

19. Thompson SC, Jolley D, Marks R. Reduction of solar keratoses by regular sunscreen use. N Engl J Med 1993;329:1147-51.

20. Ulrich C, Jürgensen JS, Degen A, et al. Prevention of non-melanoma skin cancer in organ transplant patients by regular use of a sunscreen: a 24 months, prospective, case-control study. Br J Dermatol 2009;161(Suppl 3):78-84.

21. Silva ES, Tavares R, Paulitsch F da S, et al. Use of sunscreen and risk of melanoma and non-melanoma skin cancer: a systematic review and meta-analysis. Eur J Dermatol 2018;28:186-201.

22. Rueegg CS, Stenehjem JS, Egger M, et al. Challenges in assessing the sunscreen-melanoma association. Int J Cancer 2019;144:2651-68.

23. Sunscreen FAQs. Des Plaines (IL): American Academy of Dermatology Association; 2020. Accessible ici : www.aad.org/media/stats-sunscreen (consulté le 16 août 2020).

24. Gloster HM, Neal K. Skin cancer in skin of color. Vol. 55. J Am Acad Dermatol 2006;55:741-60.

25. Kohli I, Nicholson CL, Williams JD, et al. Greater efficacy of SPF 100+ sunscreen compared with SPF $50+$ in sunburn prevention during 5 consecutive days of sunlight exposure: A randomized, double-blind clinical trial. J Am Acad Dermatol 2020;82:869-77.

26. Williams JD, Maitra P, Atillasoy E, et al. SPF $100+$ sunscreen is more protective against sunburn than SPF $50+$ in actual use: results of a randomized, doubleblind, split-face, natural sunlight exposure clinical trial. J Am Acad Dermatol 2018;78:902-910.e2.

27. Recalls and safety alerts: important sunscreen safety tips for Canadians. Ottawa: Health Canada; 2018. Accessible ici : healthycanadians.gc.ca/recall -alert-rappel-avis/hc-sc/2018/66966a-eng.php (consulté le 15 mars 2020).

28. Li H, Colantonio S, Dawson A, et al. Sunscreen application, safety, and sun protection: the evidence. J Cutan Med Surg 2019;23:357-69.

29. Cestari T, Buster K. Photoprotection in specific populations: children and people of color. J Am Acad Dermatol 2017;76:S110-21.

30. Autier P, Boniol M, Severi G, et al.; European Organization for Research and Treatment of Cancer Melanoma Co-operative Group. Quantity of sunscreen used by European students. Br J Dermatol 2001;144:288-91.

31. Petersen B, Datta P, Philipsen PA, et al. Sunscreen use and failures-on site observations on a sun-holiday. Photochem Photobiol Sci 2013;12:190-6. 
32. Neale R, Williams G, Green A. Application patterns among participants randomized to daily sunscreen use in a skin cancer prevention trial. Arch Dermatol 2002;138: 1319-25.

33. Marrett LD, Chu MBH, Atkinson J, et al. An update to the recommended core content for sun safety messages for public education in Canada: a consensus report. Can J Public Health 2016;107:e473-9.

34. Labeling and effectiveness testing: sunscreen drug products for over-thecounter human use - small entity compliance guide. Silver Spring (MD): US Food and Drug Administration; 2012. Accessible ici : www.fda.gov/regulatory -information/search-fda-guidance-documents/labeling-and-effectiveness-testing -sunscreen-drug-products-over-counter-human-use-small-entity (consulté le 16 mars 2020).

35. de Gálvez MV, Aguilera J, Buendía EA, et al. Time required for a standard sunscreen to become effective following application: a UV photography study. $J$ Eur Acad Dermatol Venereol 2018;32:e123-4.

36. Bodekær M, Akerström U, Wulf HC. Accumulation of sunscreen in human skin after daily applications: a study of sunscreens with different ultraviolet radiation filters. Photodermatol Photoimmunol Photomed 2012;28:127-32.

37. Beyer DM, Faurschou A, Philipsen PA, et al. Sun protection factor persistence on human skin during a day without physical activity or ultraviolet exposure. Photodermatol Photoimmunol Photomed 2010;26:22-7.

38. Bodekaer M, Faurschou A, Philipsen PA, et al. Sun protection factor persistence during a day with physical activity and bathing. Photodermatol Photoimmunol Photomed 2008;24:296-300

39. Stokes RP, Diffey BL. A novel ex vivo technique to assess the sand/rub resistance of sunscreen products. Int J Cosmet Sci 2000;22:329-34.

40. Stokes RP, Diffey BL. The water resistance of sunscreen and day-care products. Br J Dermatol 1999;140:259-63.

41. Diffey BL. When should sunscreen be reapplied? J Am Acad Dermatol 2001; 45:882-5.

42. Barr J. Spray-on sunscreens need a good rub. J Am Acad Dermatol 2005;52:180-1.

43. Pearce K, Goldsmith WT, Greenwald R, et al. Characterization of an aerosol generation system to assess inhalation risks of aerosolized nano-enabled consumer products. Inhal Toxicol 2019;31:357-67.

44. Rodríguez E, Valbuena MC, Rey M, et al. Causal agents of photoallergic contact dermatitis diagnosed in the national institute of dermatology of Colombia. Photodermatol Photoimmunol Photomed 2006;22:189-92.

45. Matta MK, Zusterzeel R, Pilli NR, et al. Effect of sunscreen application under maximal use conditions on plasma concentration of sunscreen active ingredients: a randomized clinical trial. JAMA 2019;321:2082-91.

46. Califf RM, Shinkai K. Filling in the evidence about sunscreen. JAMA 2019;321: 2077-9.

47. Matta MK, Florian J, Zusterzeel R, et al. Effect of sunscreen application on plasma concentration of sunscreen active ingredients: a randomized clinical trial. JAMA 2020;323:256-67.

48. Filipe P, Silva JN, Silva R, et al. Stratum corneum is an effective barrier to TiO2 and ZnO nanoparticle percutaneous absorption. Skin Pharmacol Physiol 2009;22:266-75.
49. Rai R, Shanmuga SC, Srinivas C. Update on photoprotection. Indian J Dermatol 2012;57:335-42.

50. Ghazipura M, McGowan R, Arslan A, et al. Exposure to benzophenone-3 and reproductive toxicity: a systematic review of human and animal studies. Reprod Toxicol 2017;73:175-83.

51. Buck Louis GM, Kannan K, Sapra KJ, et al. Urinary concentrations of benzophenonetype ultraviolet radiation filters and couples' fecundity. Am J Epidemiol 2014;180:1168-75.

52. Balmer ME, Buser H-R, Müller MD, et al. Occurrence of some organic UV filters in wastewater, in surface waters, and in fish from Swiss Lakes. Environ Sci Technol 2005;39:953-62.

53. da Silva CP, Emídio ES, de Marchi MRR. The occurrence of UV filters in natural and drinking water in São Paulo State (Brazil). Environ Sci Pollut Res Int 2015;22: 19706-15.

54. Ramos S, Homem V, Alves A, et al. A review of organic UV-filters in wastewater treatment plants. Environ Int 2016;86:24-44

55. Schneider SL, Lim HW. Review of environmental effects of oxybenzone and other sunscreen active ingredients. J Am Acad Dermatol 2019;80:266-71.

56. Downs CA, Kramarsky-Winter E, Segal R, et al. Toxicopathological effects of the sunscreen UV filter, oxybenzone (Benzophenone-3), on coral planulae and cultured primary cells and its environmental contamination in Hawaii and the U.S. Virgin Islands. Arch Environ Contam Toxicol 2016;70:265-88.

57. Ouchene L, Litvinov IV, Netchiporouk E. Hawaii and other jurisdictions ban oxybenzone or octinoxate sunscreens based on the confirmed adverse environmental effects of sunscreen ingredients on aquatic environments. $J$ Cutan Med Surg 2019;23:648-9.

58. Sarkar AK. An evaluation of UV protection imparted by cotton fabrics dyed with natural colorants. BMC Dermatol 2004;4:15.

59. Gies P. Photoprotection by clothing. Photodermatol Photoimmunol Photomed 2007;23:264-74

60. Jansen R, Osterwalder U, Wang SQ, et al. Photoprotection: part II. Sunscreen: development, efficacy, and controversie. J Am Acad Dermatol 2013;69:867. e1-14.

61. Wang SQ, Osterwalder U, Jung K. Ex vivo evaluation of radical sun protection factor in popular sunscreens with antioxidants. J Am Acad Dermatol 2011;65:525-30.

62. Lim HW, Arellano-Mendoza M-I, Stengel F. Current challenges in photoprotection J Am Acad Derm 2017;76(3S1):S91-9.

63. Choudhry SZ, Bhatia N, Ceilley R, et al. Role of oral Polypodium leucotomos extract in dermatologic diseases: a review of the literature. J Drugs Dermatol 2014;13:148-53.

64. Nestor MS, Berman B, Swenson N. Safety and efficacy of oral polypodium leucotomos extract in healthy adult subjects. J Clin Aesthet Dermatol 2015;8:19-23.

65. Middelkamp-Hup MA, Pathak MA, Parrado C, et al. Oral Polypodium leucotomos extract decreases ultraviolet-induced damage of human skin. J Am Acad Dermatol 2004;51:910-8

66. Chen AC, Martin AJ, Choy B, et al. A phase 3 randomized trial of nicotinamide for skin-cancer chemoprevention. N Engl J Med 2015;373:1618-26.
Intérêts concurrents : Toni Burbidge signale avoir reçu des honoraires d'AbbVie, de Celgene, de Janssen, de LEO Pharma et de Lilly. Aucun autre intérêt concurrent n'a été déclaré.

Cet article a été révisé par des pairs.

Affiliations : Département de médecine (Megan Sander, Burbidge), Section de dermatologie et École de médecine Cumming (Michael Sander), Université de Calgary, Calgary, Alb.; Division de dermatologie (Beecker), Service de médecine, Hôpital d'Ottawa; Faculté de médecine (Beecker), Université d'Ottawa et Institut de recherche de l'Hôpital d'Ottawa (Beecker), Ottawa, Ont.

Collaborateurs : Tous les auteurs ont contribué à l'élaboration et à la conception des travaux ainsi qu'à l'acquisition, à l'analyse et à l'interprétation des données. Tous les auteurs ont participé à la rédaction du manuscrit, en ont révisé de façon critique le contenu intellectuel important, ont donné leur approbation finale pour la version destinée à être publiée, et assument l'entière responsabilité de tous les aspects du travail.

Propriété intellectuelle du contenu : Il s'agit d'un article en libre accès distribué conformément aux modalités de la licence Creative Commons Attribution (CC BY-NC-ND 4.0), qui permet l'utilisation, la diffusion et la reproduction dans tout médium à la condition que la publication originale soit adéquatement citée, que l'utilisation se fasse à des fins non commerciales (c.-à-d., recherche ou éducation) et qu'aucune modification ni adaptation n'y soit apportée. Voir : https://creativecommons. org/licenses/by-nc-nd/4.0/deed.fr.

Correspondance : Megan Sander, msander@ucalgary.ca 